\title{
Grid Connection Improvements by Control Strategy Selection for Wave Energy Converters
}

\author{
M. Santos ${ }^{1}$,E. Tedeschi ${ }^{2}$, P. Ricci ${ }^{1}$, M. Molinas ${ }^{2}$ and J. L. Martin ${ }^{3}$ \\ ${ }^{1}$ Tecnalia \\ Parque Tecnológico, E202, \\ 48170 Zamudio (Spain) \\ Phone/Fax number : +34 99 6002266/99 e-mail: maider.santos@tecnalia.com, pierpaolo.ricci@tecnalia.com \\ ${ }^{2}$ Department of Electric Power Engineering \\ Norwegian University of Science and Technology \\ 7491 Trondheim (Norway) \\ Phone/Fax number:+47 735942 24/79, e-mail: elisabetta.tedeschi@elkraft.ntnu.no, marta.molinas@elkraft.ntnu.no \\ ${ }^{3}$ Department of Electronics and Telecommunications \\ University of the Basque Country \\ 48013 Bilbao (Spain) \\ Phone/Fax number:+34 94601 7351/4259, e-mail: joseluis.martin@ehu.es
}

\begin{abstract}
In this paper a wave-to-wire approach to model the exploitation of ocean energy by point absorbers in heave is presented. Attention is focused on the impact that the control strategy of the Wave Energy Converter (WEC) has both on the power performance of the single device and on the grid power quality at the connection point, when point absorbers are arranged in wave farms. Two different control strategies are proposed and compared to theoretical ones such as the complex-conjugate method. Their effectiveness in improving the system overall power extraction while reducing each Power Take-Off's rating and easing the Wave Farm grid integration is proved by time domain simulations, developed both at singledevice level and at farm level.
\end{abstract}

\section{Key words}

Wave Energy Converters, grid connection, control strategies, point absorbers, Wave Farms.

\section{Introduction}

Despite the increasing attention paid to Wave Energy Converters (WECs), a single leading technology has not yet been established. One of the most promising concepts is that of point absorbers, due to their reduced infrastructural costs and suitability for exploitation in large wave farms.

Point absorbers have been extensively studied in the past decades, with special focus on hydrodynamics, survivability and control issues, to find possible optimizations at single-device level. In order to obtain commercially viable solutions, however, it is now mandatory to achieve the grid connection of such WECs, mainly arranged in arrays. To take this final step it is fundamental not only the rational design of the Power Take-Off (PTO) for the single WEC, but also the careful analysis of the effect that a medium/large Wave Farm has on the power system, whenever connected. The goal of this paper is to show how different control techniques can severely affect the overall power conversion process, including the grid connection stage.
More specifically, two straightforward control strategies for point absorbers in heave will be proposed and analyzed in order to prove their usefulness in limiting the size of the required PTO and easing the WEC grid integration, while improving the total average power extraction.

\section{System modelling}

The considered system is composed by a cylindrical point absorber in heave, i.e. a single degree of freedom buoy, as the one schematically depicted in Fig. 1. Its main parameters are reported in Table I. The point absorber is directly connected to an electrical generator, without any intermediate hydraulic or pneumatic stage. Each WEC is also equipped with a full power converter allowing both the generator control and the grid interconnection.

\section{A. Hydrodynamic model of the system}

For the solution of the hydrodynamic problem, linear water wave theory is applied, based on the hypotheses of incompressible irrotational flow and inviscid fluid. This allows to apply boundary element methods and compute the velocity potential in its components (radiated and

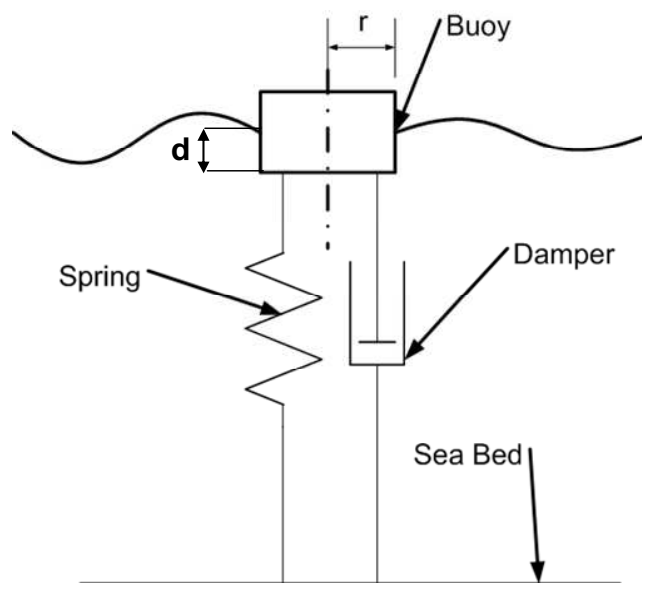

Fig.1. Schematic model of the considered point absorber (buoy) 
diffracted wave fields) and obtain the hydrodynamic coefficients.

On a general approach the equation of motion for a single body oscillating in heave is:

$m \ddot{x}=F_{e}+F_{r}+F_{h}+F_{\text {PTO }}$

where $x$ and $m$ are buoy position and mass, respectively. $F_{e}$ is the waves excitation force and $F_{r}$ the radiation force. $F_{h}$ represents the buoyancy effect and $F_{\text {PTO }}$ the PTO control force. To take into account nonlinearities, particularly when they can be modelled as time-varying coefficients of a system of Ordinary Differential Equations (ODEs), it is useful to apply a time-domain model based on the Cummins equation [1], whose use is widespread in seakeeping applications. This is based on an integro-differential equation involving convolution terms that account for the radiation forces. For the case of a single body floating in heave, the Cummins equation can be expressed as:

$\left(m+a_{\infty}\right) \ddot{x}(t)+\int_{-\infty}^{t} K_{r a d}(t-\tau) \dot{x}(\tau) d \tau+\rho g S x(t)+F_{e x t}(x, \dot{x}, t)=F_{e}(t)$

where $\mathrm{a}_{\infty}$ is the added mass at infinite frequency, $g$ is the gravity constant, $\rho$ the water density and $S$ the buoy surface. $K_{\text {rad }}(t)$ is the radiation impulse response function, representing a memory effect due to the radiation forces originated by the past motion of the body.

In this formulation all the possible nonlinearities are included in the term $F_{\text {ext }}$, which represents the external forces applied to the system due, for example, to the PTO or to the moorings and could be possibly linked to other independent variables that form a set of ODEs [2].

The hydrodynamic parameters like added mass and damping have been obtained using a boundary-element code and the convolution term has been represented as a polynomial transfer function obtained through a frequency-domain identification method [3].

\section{Analysis in regular and irregular waves}

As a preliminary evaluation of the expected system performance, an analysis under sinusoidal waves is carried out. Two classical theoretical control strategies for wave power absorption are passive loading and complex-conjugate control [4], whose performances are well-established in regular waves.

Passive loading implies that the PTO applies a pure damping to the point absorber, i.e. a force which is proportional to its velocity. In this case, both, instantaneous and average extracted power, are lower than in the case of complex-conjugate control, but the power flow is unidirectional.

Table I. - Data of the selected simulation test case (reg. waves)

\begin{tabular}{|c|c|c|c|}
\hline Quantity & Symbol & $\begin{array}{c}\text { Unit of } \\
\text { measure }\end{array}$ & Value \\
\hline Design wave amplitude & $A$ & {$[\mathrm{~m}]$} & 1 \\
\hline Design wave period & $T$ & {$[\mathrm{~s}]$} & 6.25 \\
\hline Buoy radius & $r$ & {$[\mathrm{~m}]$} & 5 \\
\hline Buoy draft & $d$ & {$[\mathrm{~m}]$} & 5 \\
\hline Buoy mass & $m$ & {$[\mathrm{Kg}]$} & 402520 \\
\hline $\begin{array}{c}\text { Added mass at } \\
\text { considered frequency }\end{array}$ & $a$ & {$[\mathrm{Kg}]$} & 222750 \\
\hline Spring stiffness & $K$ & {$[\mathrm{~N} / \mathrm{m}]$} & 789740 \\
\hline Total buoy damping & $B$ & {$[\mathrm{Kg} / \mathrm{s}]$} & 52872 \\
\hline
\end{tabular}

Complex-conjugate control aims at creating a resonance condition between the point absorber motion and the incident waves, so that the average power extraction is maximized. In this case, the force that is applied by the PTO has a component proportional to the buoy velocity and another to the buoy position. Its main drawbacks are the bi-directional power flow and the high values reached by the instantaneous power.

The system expected performance when applying these two control strategies has been evaluated with reference to a sinusoidal design wave having period $T=6.25 \mathrm{~s}$ and amplitude, $A=1 \mathrm{~m}$. Under such assumptions, application of passive loading leads to extract, as a maximum, an average power $P_{\text {avg }}=114.35 \mathrm{~kW}$ and the peak power doubles the average one.

By applying complex-conjugate control an average power of $P_{\text {avg }}=242.50 \mathrm{~kW}$ can be extracted, but in this case the maximum power is also increased to $P_{\max }=1 \mathrm{MW}$.

In order to gain a more precise insight into the system operations, the two control strategies have been also tested under irregular waves. To obtain comparable conditions between regular and irregular waves, a criterion of "equal energy period" (3.a) and "equal energy transport" (3.b) was applied. Thus, the significant wave height, $H_{s}$, and energy period, $T_{e}$, corresponding to the sinusoidal design wave considered above, have been selected according to:

$T_{e}=T$

$E_{\text {sin }}=\frac{\rho g}{2} A^{2}$

$\left.E_{\text {irr }}=\frac{\rho g}{16} H_{s}{ }^{2}\right\} E_{\text {sin }}=E_{\text {irr }} \rightarrow H_{s}=2 \sqrt{2} A$

From the corresponding Bretschneider wave energy spectrum [5], irregular waves profiles were created and corresponding excitation forces acting on the point absorber were derived from the Haskind relations [3].

The control coefficients corresponding to the previous optimization of both complex-conjugate control and passive loading under regular waves were then applied, as constant parameters, to a corresponding irregular waves profile.

Under such conditions passive loading gives an average extracted power of $P_{a v g}=66.77 \mathrm{~kW}$, with a peak power of $P_{\text {max }}=699.65 \mathrm{~kW}\left(P_{\max } / P_{\text {avg }}=k=10.5\right)$, while complexconjugate control application results in an average power extraction of $P_{\text {avg }}=90.92 \mathrm{~kW}$ with a maximum value of $P_{\max }=1.6763 \mathrm{MW}(k=19.4)$. The extremely high value of the peak to average power ratio $(k)$ in the case of complex-conjugate control makes this control strategy unfeasible from the PTO rating standpoint. Therefore, in the following, only control strategies including a pure

Table II. - Constant passive loading optimization

\begin{tabular}{|c|c|c|c|c|}
\hline $\mathrm{R}_{\mathrm{L}}(\Omega)$ & $j$ & $\mathrm{P}_{\text {avg }}[\mathrm{kW}]$ & $\mathrm{P}_{\max }[\mathrm{kW}]$ & $k$ \\
\hline 181924.6 & 1.1 & 67.598 & 703.78 & 10.41 \\
\hline 198463.2 & 1.2 & 68.156 & 703.64 & 10.32 \\
\hline 215001.8 & 1.3 & 68.497 & 700.20 & 10.22 \\
\hline 231540.4 & 1.4 & 68.659 & 694.24 & 10.11 \\
\hline 248079.0 & 1.5 & 68.671 & 686.36 & 9.99 \\
\hline 264617.6 & 1.6 & 68.559 & 677.04 & 9.87 \\
\hline 281156.2 & 1.7 & 68.343 & 666.65 & 9.75 \\
\hline
\end{tabular}


damping component will be considered and analyzed.

As a first step to improve the WEC power performance, it is important to verify if a constant damping parameter $\left(R_{L}\right)$ being different from the one that is optimal under (corresponding) sinusoidal conditions $\left(R_{\text {Lref }}=165.4 \mathrm{k} \Omega\right.$ ) can lead to an increased average power extraction, while still limiting the peak power to reasonable values. A specific trial and error test was performed in the selected irregular wave condition. Results are reported in Table II. They show that a damping parameter increased of $50 \%$ ( $j=R_{L} / R_{\text {Lref }}$ ) with respect to the reference one, ensures the highest average power extraction of $P_{\text {avg }}=68.67 \mathrm{~kW}$, with a reduced $k=10$. Corresponding instantaneous power profile is reported in Fig.2. Such operating condition, shadowed in Table II, will be considered in the following as the "best (constant) passive loading" and used as a reference to evaluate other control strategies.

\section{Electrical optimization issues}

Like other renewable energies, wave energy is characterized by high intermittency and variability. This directly reflects on the power that can be delivered to the electric grid, which presents a strongly irregular profile, being a major obstacle for the integration of WECs into the local power system [6]. Thus, a number of different factors must be taken into account in order to make the grid connection feasible. They include both the rational design of the PTO, which is required for control strategy implementation, and grid connection issues related to the specific analysis of local Grid Codes and grid parameters at the Point of Common Coupling (PCC).

\section{A. Power Take-Off}

When designing the electric machine and power electronics interface allowing both the power extraction from the sea and its regulated injection into the power system, operating conditions of the WECs must be carefully taken into account. The rating of the PTO depends on the maximum power that the system must handle and is extremely influenced by the selected control strategy, as shown in Section 2.

Another crucial aspect for PTO design relates to its efficiency. Both electrical machines and power converters have an efficiency that is strongly affected by working conditions as loading factor and actual speed in the case of the electrical machine. When the PTO is largely oversized with respect to the average power extraction, due to the need to cope with large power fluctuations, it is forced to work mainly in low load conditions, thus making the overall power conversion less effective.

It is worth noting that, in the case of control strategies adopting a reactive component and if no storage system is included, the PTO must be fully bi-directional, i.e. it must allow a reversed power flow, from the grid to the point absorber, in some instants of the period, thus possibly resulting in more expensive (although more flexible) solutions.

In the following, it will be assumed that each WEC is equipped with an electrical generator and a full converter. It will be proved how a reduction in the peak to average extracted power ratio, obtained by suitable passive control techniques, can address all these issues, reducing the PTO rating and consequently improving the conversion efficiency and average power extraction.

\section{B. Grid connection}

The implementation of medium/large wave farms poses further challenges to the WECs optimization process.

The first aspect to be considered is the impact of the Wave Farm connection on the power quality of the local grid. This is affected both by the Wave Farm operating conditions and by the characteristics of the power system itself. It is expected that many of the Wave Energy Farms will be connected to the distribution system close to shore, being generally a weak grid, due to large impedance value. This can make grid connection even more critical especially in the case of large plants.

The feasibility of grid integration must be evaluated case by case, establishing the minimum power quality requirements to be ensured at the $\mathrm{PCC}$ also based on the compliance to local Grid Codes.

\section{Proposed control strategies}

In the following, two different control strategies, which are based on the measured velocity of the point absorber and take into account the actual power rating of the PTO, are presented.

\section{A. Threshold control}

Threshold control is based on the idea that whenever the instantaneous power extracted by the point absorber approaches the PTO power limit, the damping resistance must be decreased with respect to its initial value in order to avoid exceeding the power limit itself.

In this case the buoy velocity, $v$, is the reference variable to tune the control resistance, that is changed in few discrete steps, whenever each pre-specified velocity threshold is achieved.

If the reference damping is assumed to be the best one for passive loading application $\left(R_{\text {Lpass }}=248.1 \mathrm{k} \Omega\right.$, Section 2$)$ and the peak power limit is assumed to be $P_{\text {lim }}=500 \mathrm{~kW}$, the corresponding limit velocity for the point absorber results:

$$
v_{\lim }=\sqrt{\frac{P_{\lim }}{R_{\text {Lpass }}}}=1.42 \mathrm{~m} / \mathrm{s}
$$

Accordingly, the proposed algorithm for the threshold control is:

$$
\begin{array}{ll}
\text { if } v<v_{\lim } & \rightarrow R_{L}=R_{\text {Lpass }} \\
\text { if } v_{\lim }<v<\sqrt{2} v_{\lim } & \rightarrow R_{L}=R_{\text {Lpass }} / 2 \\
\text { if } \sqrt{2} v_{\lim }<v<\sqrt{3} v_{\lim } & \rightarrow R_{L}=R_{\text {Lpass }} / 3
\end{array}
$$

This specific coordinate selection of damping reduction criterion and velocity thresholds is made in order to ensure that the peak power limit is strictly respected, as can be seen from the blue line in the detail of Fig. 3. The control technique has been tested with the same irregular wave profile used in Section 2 for passive loading optimization, having $H_{s}=2.82 \mathrm{~m}$ and $T_{e}=6.25 \mathrm{~s}$. The average extracted power is in this case $P_{\text {avg }}=69.03 \mathrm{~kW}$, and the peak to average power ratio is $k=7.24$. 


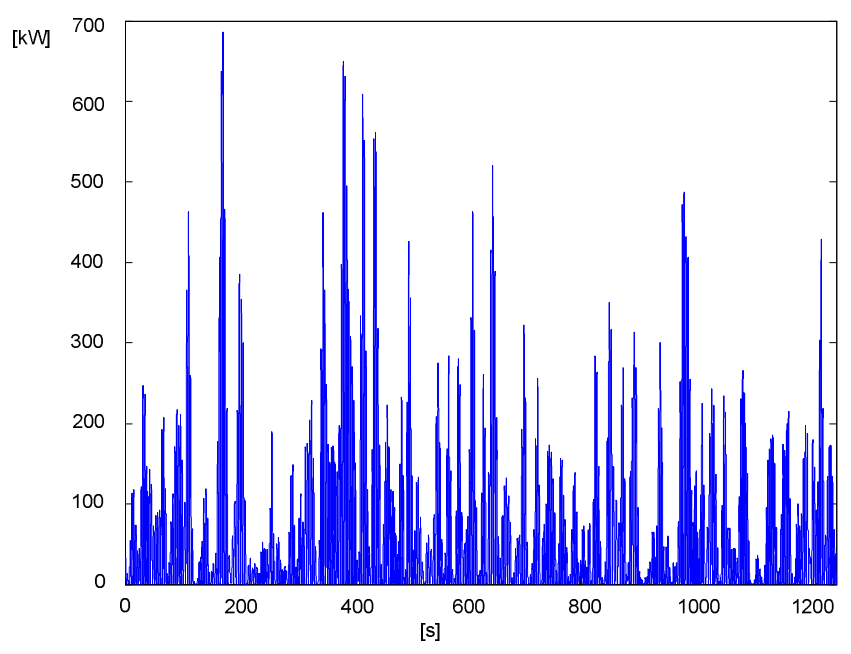

Fig. 2. Instantaneous extracted power when applying the best (constant) passive loading with the selected irregular wave profile having $\mathrm{Hs}=2.82 \mathrm{~m}$ and $\mathrm{Te}=6.25 \mathrm{~s}$

A consistent reduction in the PTO rating (-27\%) can therefore be achieved while extracting almost the same average power $(+0.5 \%)$ with respect to the best constant passive loading case.

\section{B. Equivalent saturation control}

The goal of the second control strategy is to ensure the same advantages as the threshold control in terms of peak power limitation and average power extraction, while avoiding the abrupt changes in the damping parameter that are intrinsic in the previous technique.

The peak power limit is still assumed to be $P_{\text {lim }}=500 \mathrm{~kW}$ and the point absorber velocity remains the reference value for control tuning.

In this case the control technique behaves as an equivalent saturation [7-8] (thick trapezoidal waveform in the zoom of Fig. 3) and the corresponding control algorithm is very straightforward:

$$
\begin{array}{ll}
\text { if } v<=v_{\text {lim }} & \rightarrow R_{L}=R_{\text {Lpass }} \\
\text { if } v>v_{\text {lim }} & \rightarrow R_{L}=P_{\text {lim }} / v^{2}
\end{array}
$$

where $R_{\text {Lpass }}$ is the same as in the previous cases and $v_{\text {lim }}$ is still expressed by (4).

The instantaneous power resulting from the application of such control technique, with the same irregular profile tested before, is also reported in Fig. 3. In this case, the average extracted power is $P_{a v g}=68.72 \mathrm{~kW}$, with a peak to average power ratio $k=7.27$.

It can be noted that equivalent saturation control still enhance the power performance with respect to passive loading and the average extracted power is only slightly inferior to the one obtained by using the threshold control. This is due to the different impact that the two techniques have on the point absorber's velocity and depends on the specific irregular wave profile.

The smoother damping variations of the equivalent saturation control, however, may enhance both the mechanical and electrical performances of the PTO, by reducing its stress, thus justifying the adoption of such control technique despite the minimal decrease in the average power.

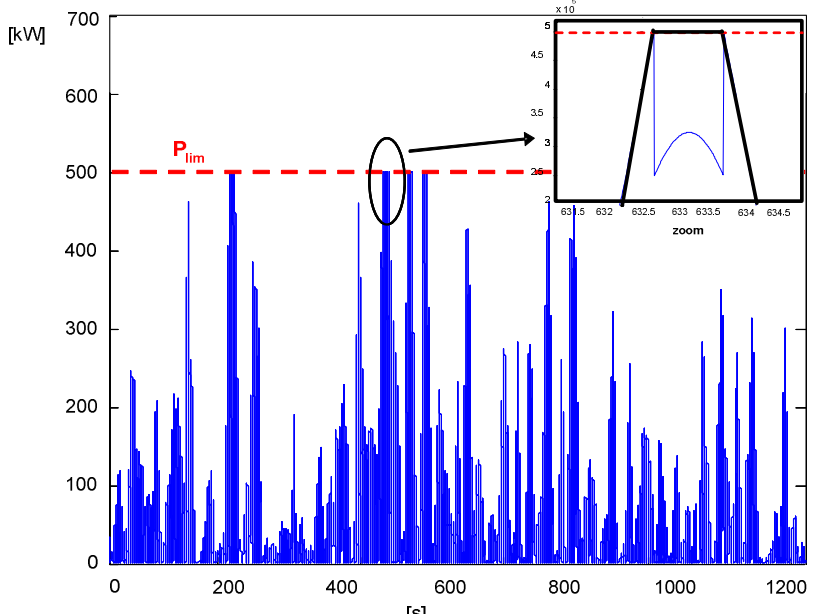

Fig. 3. Instantaneous extracted power when applying the threshold control (blue line in the zoom) and the equivalent saturation control (black line in the zoom) with the selected irregular wave profile having $\mathrm{Hs}=2.82 \mathrm{~m}$ and $\mathrm{Te}=6.25 \mathrm{~s}$

A final issue regarding the equivalent saturation control relates to the proper selection of the saturation level. A specific test in the considered sea state has been performed in order to evaluate how the average power extraction is affected by the selected saturation level. As can be seen from Fig 4.a, limited average power losses (within $10 \%$ in the shadowed area) could be achieved even with a saturation level that is more than halved with respect to the selected $500 \mathrm{~kW}$ level. Correspondingly, Fig. 4.b shows that the peak to average power ratio can be decreased till around 3.5, while staying within such $10 \%$ average power loss.
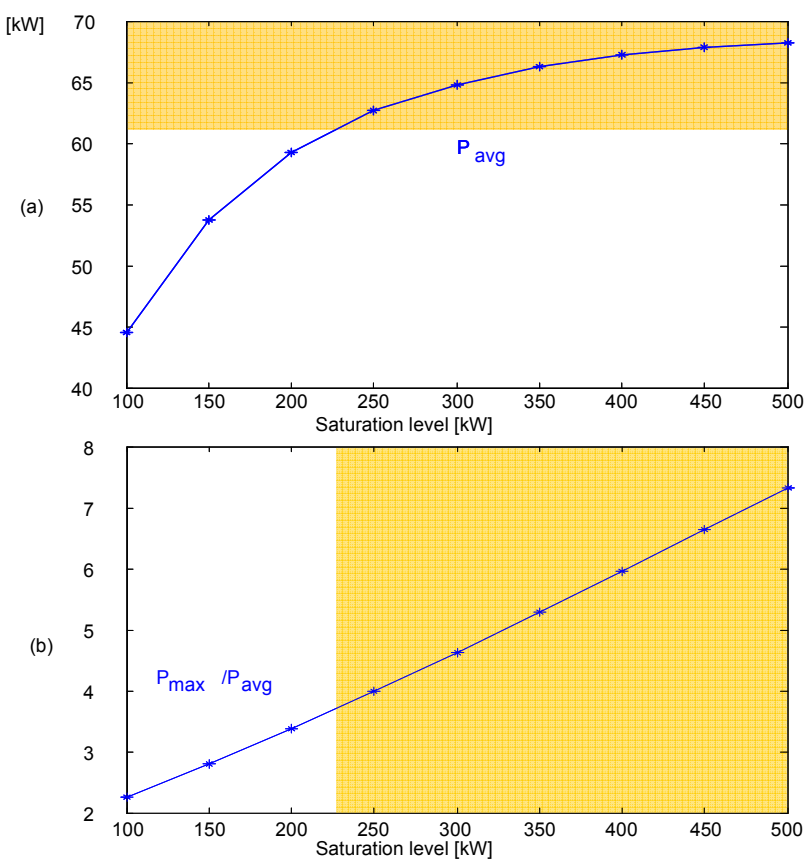

Fig. 4. Impact of saturation level selection

The reason why an higher power saturation level $\left(P_{\max }=500 \mathrm{~kW}\right)$ was kept in this study was to ensure the exploitability of the system also in more energetic sea states, avoiding a consistent limitation of the achievable power performance under different conditions. 
However, the proposed considerations on the effectiveness of equivalent saturation technique in reducing the potential rating of the PTO with limited average power loss hold in general, irrespective of the specific sea state.

\section{Global power performance assessment}

In order to evaluate the beneficial effect of the proposed control strategies on the grid integration and to compare them to the traditional passive loading solution, it is assumed, as a first case, that a Wave Farm, including 28 WECs as the one of Fig. 1, is considered.

A $0.69 / 13.2 \mathrm{kV}$ transformer is used every 7 WECs to convey the power to 4 subsea offshore cables, having kilometric impedance: $r+j x=0.124+j 0.1 \Omega$ and whose lengths vary between 3 and $6 \mathrm{~km}$, as reported in Fig. 5 . Onshore, subsea cables are connected to four identical 1 $\mathrm{km}$ over-head lines, having impedance: $r+j x=0.125+j$ $0.094 \Omega$.

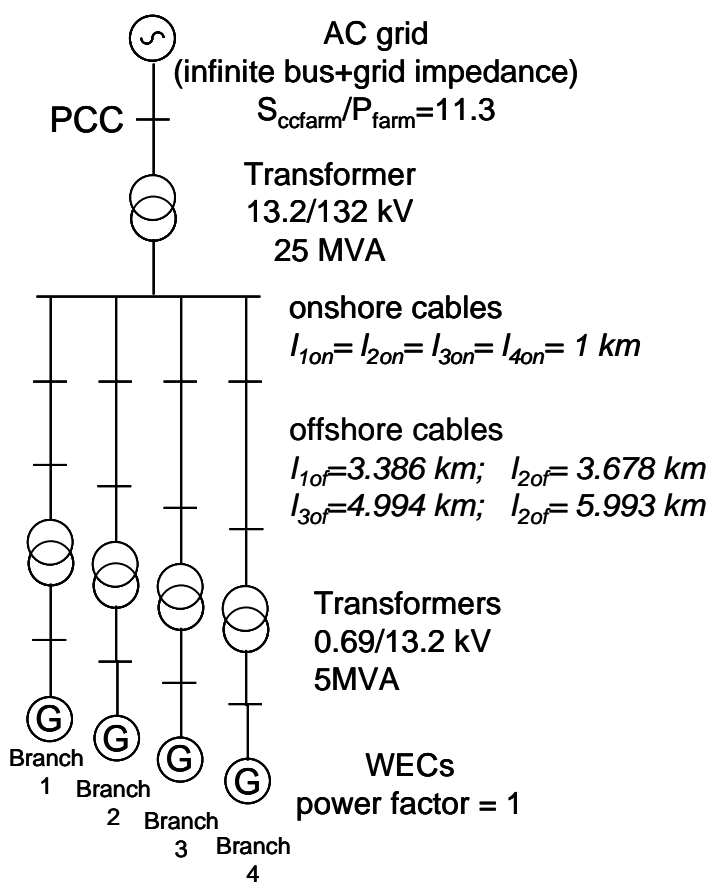

Fig. 5. Schematics of the considered Wave Farm and connection to the AC Power Grid.

One 13.2/132 kV transformer is then used for the Wave Farm connection to the PCC, being a very weak grid, with $S_{\text {ccfarm }} / P_{\text {farm }}=11.3$. Each PTO is controlled to inject the power into the local grid with a unity power factor at the WEC connection point, so that the reactive power exchange between the farm and the power system is only due to internal infrastructures (cables, transformers ...) of the farm.

Any smoothing effect due to the aggregation of many WECs is here neglected, thus assuming that the wave front reaches all the WECs at the same time, in order to perform a worst case analysis under the specified conditions.

A detailed DiGSILENT ${ }^{\circledR}$ model of the considered Wave Farm has been developed in order to evaluate the voltage drop experienced at the PCC as a consequence of the Wave Farm connection and the power performance of the farm.
As a first case (Case 1 in Table III) it is assumed that each of the 28 WECs applies the best constant passive loading. Based on the data in Table II, each WEC is rated $700 \mathrm{~kW}$, resulting in a 19.6 MW Wave Farm. In this case the total average power that is extracted from the farm is $P_{\text {farm }}=1.91 \mathrm{MW}$. It can be seen from Fig. 6 that a voltage drop around $2.2 \%$ can be expected at the PCC as a consequence of the extremely varying injected power and of the high peak to average power ratio. Corresponding active and reactive power absorption at the PCC are reported in Fig. 9.a.

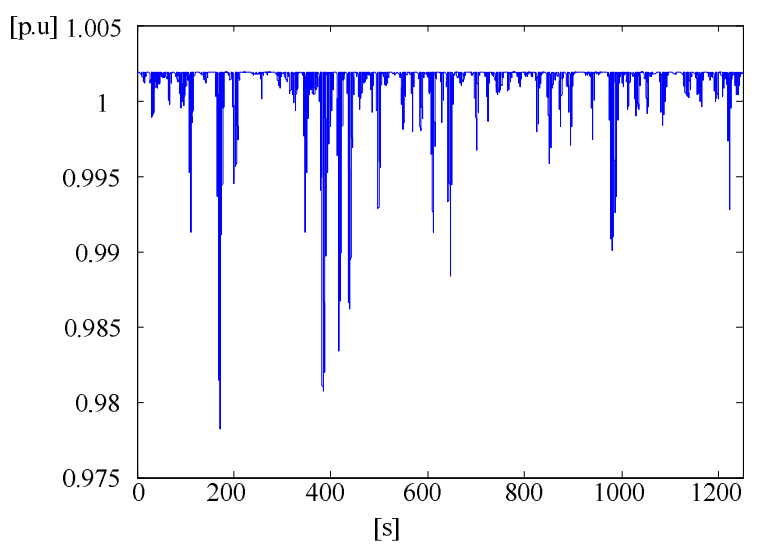

Fig. 6. Normalized voltage at the PCC due to the connection of a 19.6 MW farm with all the 28 WECs applying best onstant passive loading.

As a second case (Case 2 in Table III) it is assumed that the Wave Farm is still composed by 28 WEC, but the maximum power that can be handled by each WEC is limited to $P_{\text {lim }}=500 \mathrm{~kW}$. Thus, the Wave Farm size is reduced to $14 \mathrm{MW}$. In this case, the equivalent saturation control described in Section 4.B is applied and each WEC is still controlled to have a unity power factor at its connection point.

The average power injected into the power grid is still $P_{\text {farm }}=1.91 \mathrm{MW}$, with the instantaneous active and reactive power profiles reported in Fig. 9.b, but the PCC voltage drop never exceeds $1 \%$, as can be seen from Fig. 7.

If the $2.2 \%$ voltage drop at the PCC experienced in Case 1 is considered acceptable, the proposed equivalent saturation control can still be useful. In fact it is possible to increase the installed power of the farm, while still limiting the peak power of each WEC by the proposed saturation technique (Case 3 in Table III), so that the impact of the farm on the power grid is not deteriorated. This can be seen by considering a Wave Farm composed by 39 WECs, with each PTO saturated at $500 \mathrm{~kW}$. The total installed power results $19.5 \mathrm{MW}$. In this case the voltage drop at PCC is still below $2.2 \%$ (Fig. 8), but the average extracted power of the farm is $2.65 \mathrm{MW}$.

Thus, a significant improvement compared to the first case, where constant passive loading was applied to a farm of comparable power rating is achieved. Active and reactive power absorption at the $\mathrm{PCC}$ is reported in Fig. 9.c. It is worth noting that equivalent advantages can be obtained by the threshold control instead of the equivalent saturation one. This clearly shows how the proposed control techniques help in both increasing the average power extraction and in mitigating the grid connection impact. 


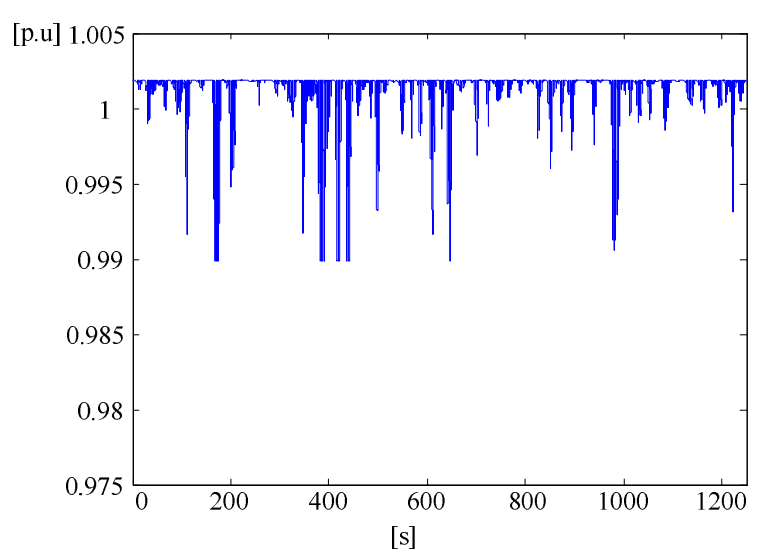

Fig. 7. Normalized voltage at the PCC due to the connection of a 14 MW farm with all the 28 WECs applying equivalent saturation control.

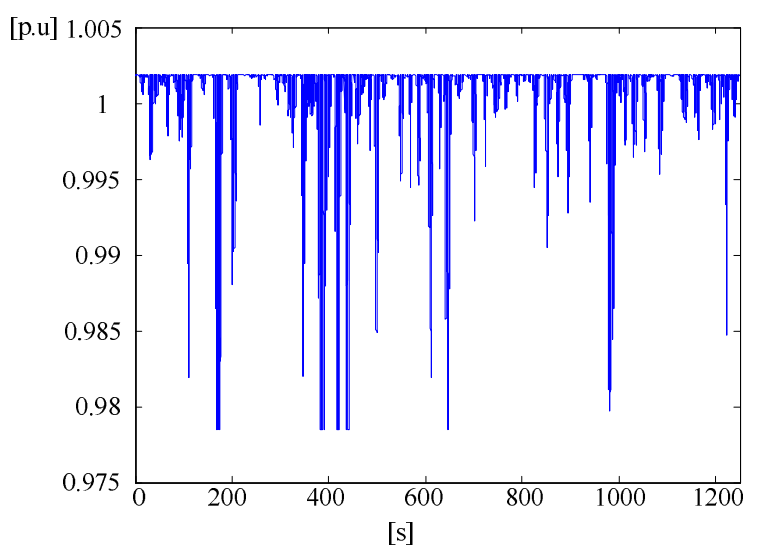

Fig. 8. Normalized voltage at the PCC due to the connection of a 19.5 MW farm with all the 39 WECs applying equivalent saturation control.

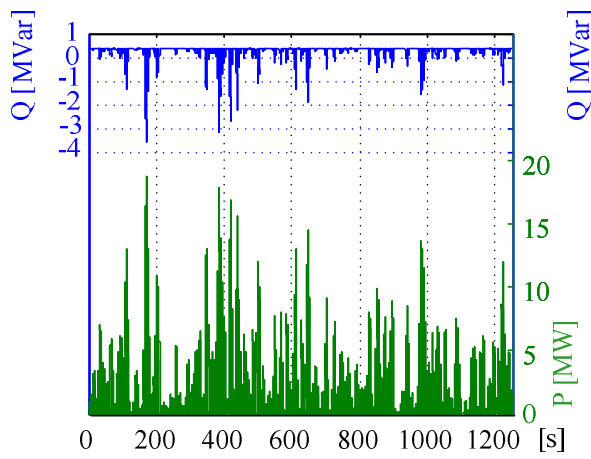

(a)

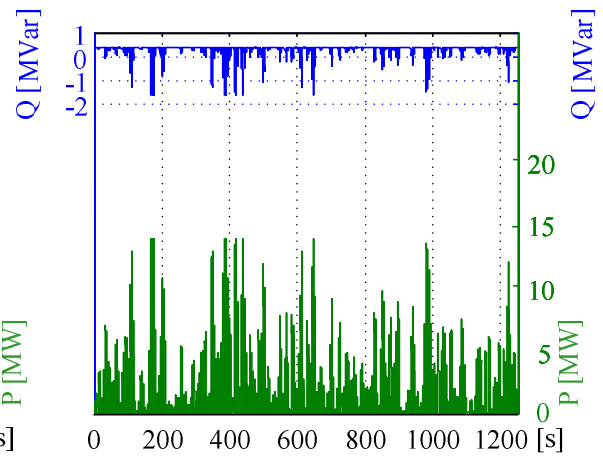

(b)

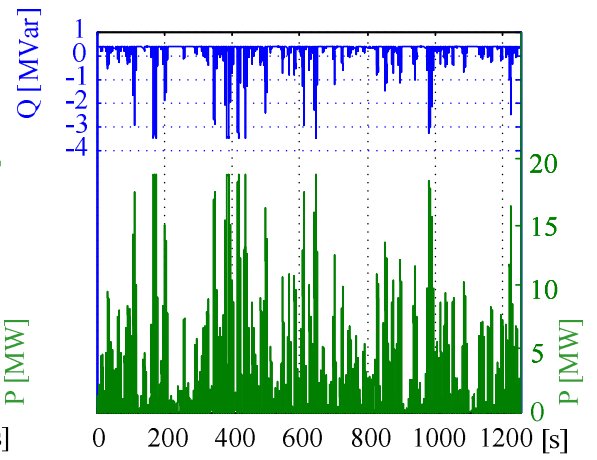

(c)

Fig. 9. Active and reactive power at the PCC in the considered case 1 (a), case 2 (b), case 3 (c).

Table III. - Data about considered Wave Farms size and performances

\begin{tabular}{|c|c|c|c|c|c|c|c|c|}
\hline & \multicolumn{4}{|c|}{ Number of WECs per branch } & $P_{\text {WEC }}$ & $P_{\text {farm }}$ rated & $P_{\text {farm }}$ avg & $\mathrm{V}_{\mathrm{PCC}} \min$ \\
\hline & Branch 1 & Branch 2 & Branch 3 & Branch 4 & $(\mathrm{~kW})$ & $(\mathrm{MW})$ & $(\mathrm{MW})$ & (p.u.) \\
\hline Case 1:p & 7 & 7 & 7 & 7 & 700 & 19,6 & 1,91 & 0,9782 \\
\hline Case 2:equiv. saturation $28 W E C s$ & 7 & 7 & 7 & 7 & 500 & 14 & 1,91 & 0,9899 \\
\hline Case 3:equiv. saturation 39WECs & 9 & 10 & 10 & 10 & 500 & 19,5 & 2,65 & 0,9784 \\
\hline
\end{tabular}

\section{Conclusion}

This paper shows how the power performances of point absorbers' Wave Farms are strongly influenced by the control strategy they apply. Two different WEC control techniques were presented and compared to most established ones in order to prove that a proper selection of control parameters helps not only in reducing each PTO ratings, but also in increasing the power extraction from the farm and enhancing the power quality at the grid connection point.

\section{Acknowledgement}

This work has been supported by a grant from Iceland, Liechtenstein and Norway through the EEA Financial Mechanism. Supported and coordinated by Universidad Complutense de Madrid.

\section{References}

[1] Cummins, W.E., "The Impulse Response Function and Ship Motions", Schiffstechnik 9 (1661), pp. 101-109. 1962

[2] P. Ricci et. al. "Control Strategies for a simple PointAbsorber Connected to a Hydraulic Power Take-off"
Proceedings of the 8th European Wave and Tidal Energy Conference, Uppsala, Sweden. 2009.

[3] T. Perez, T.I. Fossen, "Time domain versus Frequency domain Identification of Parametric Radiation Force Models for Marine Structures at Zero Speed", Modeling, Identification and Control, Vol. 29, No. 1, pp. 1-19. 2008.

[4] J. Falnes, "Ocean Wave Energy and Oscillating Systems: Linear Interaction Including Wave-Energy Extraction". Cambridge Univ. Press; 1st Ed. 2002.

[5] W. H. Michel, "Sea Spectra Revisited", Marine Technology, Vol. 36 N.4 winter 1999, pp. .211-227

[6] M. Santos, D. Ben Haim, F. Salcedo, J.L. Villate, Y.TorreEnciso, "Grid Integration of Wave Energy Farms: Basque Country Study" Proc. Of the 3rd Int. Conference on Ocean Energy (ICOE10), 6-8 October, Bilbao, Spain, pp. 1-6

[7] M. Ruellan, H.B.Ahmed, B. Multon, C. Josset, A. Babarit, A. Clement, "Design Methodology for a SEAREV Wave Energy Converter", IEEE Trans. On Energy Conversion, Vol. 25, NO.3, September 2010, pp.760-767

[8] E. Tedeschi, M. Carraro, M. Molinas, P. Mattavelli, "Analysis of Power Extraction from Irregular Waves by All-Electric Power Take-Off", Proc. Of the $2^{\text {nd }}$ IEEE Energy Conversion Congress \& Expo (ECCE10), 12-16 September 2010, Atlanta, Georgia, U.S., pp. 2370-2377 